\title{
A RIGID SPACE WHOSE SQUARE IS THE HILBERT SPACE
}

\author{
JAN J. DIJKSTRA
}

AbStract. We construct a space $X$ with only one autohomeomorphism and the property that the product $X \times X$ is homeomorphic to the separable Hilbert space.

1. Introduction. In [4] J. van Mill constructed a rigid space (i.e., a space with only one autohomeomorphism) whose square was the Hilbert cube. Since then several finite-dimensional rigid spaces with a square that is a manifold have been found; see Ancel and Singh [1] and Ancel, Duvall and Singh [2]. The examples in these papers are constructed by means of CE-decompositions of manifolds. This idea does not work if one wants to factorize the Hilbert space $l^{2}$ into rigid spaces (see Mogilski [5], who proved that any absolute retract that is a CE-image of an $l^{2}$-manifold is homeomorphic to $l^{2}$ ). Nevertheless, in this note we give a remarkably simple construction of $2^{\aleph_{0}}$ distinct rigid spaces with squares that are homeomorphic to $l^{2}$. It is also worth noting that [1, 2 and 4] use algebraic arguments, whereas our discussion is purely topological.

2. Preliminaries. In this section we have collected a few known facts related to continua. We use Sierpinski's theorem [6] that no continuum can be partitioned into countably many disjoint closed subsets. If $x$ is a point of a space $X$ then the continuum-component of $x$ is given by

$$
\mathrm{CC}(x, X)=\bigcup\{C \subset X \mid C \text { is a continuum that contains } x\} .
$$

The space $X$ is called continuum-connected if $\mathrm{CC}(x, X)=X$ for some $x$. Note that Sierpiński's theorem is also valid for continuum-connected spaces.

A closed, continuous mapping is called monotone if it is onto and has the property that the preimage of every connected set is also connected. The following lemma is due to Anderson, Curtis and van Mill [3].

Lemma. Let $B_{1}$ and $B_{2}$ be $\sigma$-Z-sets in the Hilbert cube $Q$, and let $f: Q \backslash B_{1} \rightarrow Q \backslash B_{2}$ be a homeomorphism. Then there exist a compact space $M$ and monotone mappings $\gamma_{1}, \gamma_{2}: M \rightarrow Q$ such that $\gamma_{1}^{-1}\left(B_{1}\right)=\gamma_{2}^{-1}\left(B_{2}\right)$ and $f \circ \gamma_{1}\left|\gamma_{1}^{-1}\left(Q \backslash B_{1}\right)=\gamma_{2}\right| \gamma_{2}^{-1}\left(Q \backslash B_{2}\right)$.

Received by the editors February 17, 1984.

1980 Mathematics Subject Classification. Primary 54G99.

Key words and phrases. Rigid, Hilbert space, continuum-connected.

(C)1985 American Mathematical Society $0002-9939 / 85 \$ 1.00+\$ .25$ per page 
3. The example. Let the Hilbert cube be represented by $Q=\Pi_{i=1}^{\infty}[-1,1]$, and let $\left(p_{i}\right)_{i=1}^{\infty}$ be a sequence in $(0,1)$ converging to 1 . Define, for every $i \in \mathbf{N}$, the "shrunken endface in the $i$-coordinate direction" $W_{i}$ by

$$
W_{i}=\left\{\left(x_{j}\right)_{j=1}^{\infty} \in Q \mid x_{i}=1 \text { and } x_{j} \in\left[-p_{i}, p_{i}\right] \text { for } j \neq i\right\},
$$

and put $Y=Q \backslash \cup_{i=1}^{\infty} W_{i}$. The space $Y$ was introduced by Anderson, Curtis and van Mill [3]. It is homogeneous and has the following interesting property (see [3, §3]): if $A$ is a $\sigma$-compact subset of $Y$ then $(Y \backslash A) \times(Y \backslash A)$ is homeomorphic to $l^{2}$. We construct a $\sigma$-compactum in $Y$ such that its complement is rigid.

For every $i \in \mathbf{N}$ let $Z_{i}$ be an $i$-pointed star with centre $x_{i}$ (i.e., $Z_{i}$ is the disjoint union $\left\{x_{i}\right\} \cup \cup_{j=1}^{i} J_{i j}$, where $J_{i j} \cup\left\{x_{i}\right\}$ is homeomorphic to the arc [0,1]). It is no problem to imbed the $Z_{i}$ 's pairwise disjointly in $Y$ such that the set $\left\{x_{i} \mid i \in \mathbf{N}\right\}$ is dense (imbed them, for instance, in the pseudo-interior $\prod_{i=1}^{\infty}(-1,1)$, in which case it is also obvious that every $Z_{i}$ is a $Z$-set in $Q$ ). Our example $X$ is now given by

$$
X=Y \backslash \bigcup_{i=1}^{\infty} \bigcup_{j=1}^{i} J_{i j}
$$

Observe that $P=Q \backslash X$ is a $\sigma$-Z-set in $Q$ and the $W_{i}$ 's are disjoint copies of $Q$. Since $\bigcup_{i=1}^{\infty} \bigcup_{j=1}^{i} J_{i j}$ is $\sigma$-compact it suffices to show that $X$ is rigid.

Claim. $X$ is rigid.

Proof. Note that

$$
\left\{W_{i} \mid i \in \mathbf{N}\right\} \cup\left\{J_{i j} \mid(i, j) \in \mathbf{N}^{2}, j \leqslant i\right\}
$$

forms a countable, closed covering of $P$ consisting of disjoint continuum-connected sets. According to Sierpinski, this means that it is the collection of continuumcomponents of $P$. An analogous argument yields that

$$
\mathrm{CC}\left(x_{i}, P \cup\left\{x_{i}\right\}\right)=Z_{i} \text { for } i \in \mathbf{N}
$$

and

$$
\mathrm{CC}(x, P \cup\{x\})=\{x\} \quad \text { for } x \in X \backslash\left\{x_{i} \mid i \in \mathbf{N}\right\} .
$$

This means that $x_{i}$ is the only point $x$ of $X$ whose continuum-component in $P \cup\{x\}$ contains precisely $i$ continuum-components of $P$.

Let $h$ be an autohomeomorphism of $X$. According to the lemma, there exist a compact $M$ and monotone mappings $\gamma_{1}, \gamma_{2}: M \rightarrow Q$ such that $\gamma_{1}^{-1}(P)=\gamma_{2}^{-1}(P)$ and $h \circ \gamma_{1}\left|\gamma_{1}^{-1}(X)=\gamma_{2}\right| \gamma_{2}^{-1}(X)$. Observe that if $A \subset B \subset Q$ then $A$ is a continuumcomponent of $B$ iff $\gamma_{k}^{-1}(A)$ is a continuum-component of $\gamma_{k}^{-1}(B)$. Let $x$ be a point of $X$. Since $\gamma_{1}^{-1}(\{x\})=\gamma_{2}^{-1}(\{h(x)\}) \neq \varnothing$, we have

$$
\gamma_{1}^{-1}(\mathrm{CC}(x, P \cup\{x\}))=\gamma_{2}^{-1}(\mathrm{CC}(h(x), P \cup\{h(x)\})) \text {. }
$$

Call this last set $C$ and note that $\mathrm{CC}(x, P \cup\{x\})$ contains as many continuumcomponents of $P$ as $C$ contains continuum-components of $\gamma_{1}^{-1}(P)=\gamma_{2}^{-1}(P)$. Since the same is true for $h(x)$, we have that $\mathrm{CC}(x, P \cup\{x\})$ contains precisely the same 
number of continuum-components of $P$ as $\mathrm{CC}(h(x), P \cup\{h(x)\})$. In view of the above remark, we may conclude that $h$ fixes the dense set $\left\{x_{i} \mid i \in \mathbf{N}\right\}$. This means that $h$ is the identity and, hence, $X$ is rigid.

REMARK. Note that this method yields $2^{\aleph_{0}}$ topologically distinct rigid spaces with squares homeomorphic to $l^{2}$.

\section{REFERENCES}

1. F. D. Ancel and S. Singh, Rigid finite dimensional compacta whose squares are manifolds, Proc. Amer. Math. Soc. 87 (1983), 342-346.

2. F. D. Ancel, P. F. Duvall and S. Singh, Rigid 3-dimensional compacta whose squares are manifolds, Proc. Amer. Math. Soc. 88 (1983), 330-332.

3. R. D. Anderson, D. W. Curtis and J. van Mill, A fake topological Hilbert space, Trans. Amer. Math. Soc. 272 (1982), 311-321.

4. J. van Mill, $A$ rigid space $X$ for which $X \times X$ is homogeneous; an application of infinite-dimensional topology, Proc. Amer. Math. Soc. 83 (1981), 597-600.

5. J. Mogilski, $C E$-decomposition of $l^{2}$-manifolds, Bull. Acad. Polon. Sci. Sér. Sci. Math. Astr. Phys. 27 (1979), 309-314.

6. W. Sierpiński, Un théorème sur les continus, Tôhoku Math. J. 13 (1918), 300-303.

Mathematisch InstituUt, Universiteit van Amsterdam, Roetersstraat 15, Amsterdam, The NETHERLANDS

Current address: Department of Mathematics, University of Florida, Gainesville, Florida 32611 